ORIGINAL ARTICLE

\title{
Epidemiological Profile and Clinical Experience of Abdominal Wall Hernia in Tertiary Care Hospital, Pakistan
}

\author{
UMAR REHMAN ${ }^{1}$, WARDAH MUMTAZ², FAZAL-E-NAUMAN ${ }^{3}$, ZAHID SAEED $^{4}$, ARSHID MAHMOOD ${ }^{5}$ \\ 1,2 House Officer, Pakistan Institute of Medical Sciences (PIMS), Islamabad \\ ${ }^{3}$ Assistant Professor Surgery, Islam Medical College, Sialkot \\ ${ }^{4}$ Assistant Professor of Surgery, ${ }^{5}$ Associate Professor of Surgery, Mohi ud Din Islamic Medical College, Mirpur Azad Kashmir \\ Corresponding author: Dr. Arshid Mahmood, Email: arshid-mahmood@hotmail.com, Cell No: +92 3345793149
}

\begin{abstract}
Background and Aim: Abdominal wall hernia is a communal surgical intervention complication with groin hernia being the most commonly presented hernia around the world. Delay in addressing these complications may cause morbidity and mortality. Hernias can be repaired using a variety of surgical techniques. The purpose of the current study was to assess the epidemiological profile and clinical experience of abdominal wall hernia in tertiary care hospital, Pakistan.

Materials and Methods: This epidemiological prospective study was conducted on 226 patients with abdominal wall hernia at HBS General Hospital, Islamabad for period of one year i.e from July 2020 June 2021. All the hernia patients admitted to the surgery department for abdominal wall hernias during the study period were examined. The relevant details of each individual were gathered from hospital medical and clinical records and noted in pre-designed proforma. Epidemiological and clinical profiles were tabulated and analyzed.

Results: The overall mean age of patients was $44.6 \pm 5.6$ with age ranges from 16 years to 72 years. Out of all the operated hernia patients, about 174 (77\%) were male and $52(23 \%)$ were female. The male to female ratio was 3.34:1. Of the total 226 abdominal wall hernia patients, 202 (89.4\%) patients were operated on. The prevalence of emergency-based operated hernia patients was 40 (19.8\%). The most common operated abdominal wall hernia was groin hernia in $162(80.4 \%)$ followed by umbilical, epigastric, paraumbilical, and rarer hernia with respective prevalence $20(9.9 \%), 8(3.9 \%), 10(4.9 \%)$, and $2(0.9 \%)$. Ascites, prostatic problems, chronic cough, previous surgeries, chronic constipation, and obesity were the different predisposing factors. The prevalence of predisposing factors were ascites in $3(1.3 \%)$, prostatic problems $46(20.4 \%)$, chronic cough 113 $(50 \%)$, previous surgeries $20(8.8 \%)$, chronic constipation $41(18.4 \%)$, and obesity $3(1.3 \%)$.

Conclusion: Our study found that the male population is more susceptible to abdominal wall hernia compared to the female population. Low socioeconomic status with major risk factors such as constipation, old age, lifting heavy objects, smoking, surgery phobia, homeopathy medicine treatment were the main factors for increased rate of morbidity and mortality.

Keywords: Abdominal wall hernia; epidemiology; risk factors
\end{abstract}

\section{INTRODUCTION}

Abdominal wall hernia repair accounts for $16-19 \%$ of surgical interventions in the surgical units causing morbidity and mortality among patients of developing countries [1,2]. Inguinal hernias are the most common type of hernia, accounting for approximately 7 in 10 cases of all abdominal wall hernias [3]. The incidence of inguinal hernia is 175 per 100,000 people [4]. However, only one-third of these are surgically repaired. In developing countries, patients' delayed clinical presentation is a major challenge in surgical practice and scarce of privately sponsored health care facilities. This obliges the balance between costly cutting-edge and reasonable surgical practice [5]. An abdominal wall hernia is an originally generated sac protruding through the covering of the abdominal musculoaponeurotic [6]. The most common type of hernia is a Groin hernias whereas inguinal hernias is more significant than femoral hernias. The prevalence of the most common abdominal wall hernia is $1.7 \%$ for patients of all age and $4 \%$ for above 45 years which lead to a higher incidence of abdominal wall hernia in older age patients [7]. The risk of mortality in men and women is $27 \%$ and $3 \%$ respectively due to inguinal hernia which develops $75 \%$ abdominal wall hernia $[8,9]$.
It is estimated that about 20 million hernias are repaired annually around the globe. Umbilical, epigastric, paraumbilical, incisional, traumatic, and Spigelian are the other types of hernia. Women usually suffer from femoral hernia while men from inguinal hernia [10]. The common hernias of childhood age areumbilical and inguinal hernia. The primary means of hernia diagnosis is a clinical examination with symptomatology. However, radiography to diagnose hernia might be required. The preferred repair method for lowering recurrence rate is Meshrepair however, herniorrhaphyisa is the most economical option for most patients [11]. The preferred procedure for pediatric age hernia patients is Mayo's repair and herniotomy. Laparoscopic surgery has grown in popularity due to itslower morbidity rate and postoperative rapid recovery to a normal routine, but it is expensive in local settings. Postoperative morbidity and mortality are increased by delayed presentations [12]. Simultaneous efforts to conduct area-specific surveys are required, educate the commonalities, and cut costs, allowing for early diagnosis and provision best available techniques to strata of society. The goals of this study were to assess the clinical burden and profile of hernia patients in a tertiary care hospital, as well as to describe our experience treating them. 


\section{MATERIALS AND METHODS}

This epidemiological prospective study was conducted on 226 patients with abdominal wall hernia admitted at Surgery department of HBS General Hospital, Islamabad from January 2021 to October 2021. All the patients admitted during the study period for abdominal wall hernias were examined. The relevant details of each individual were gathered from hospital medical and clinical records and noted in pre-designed proforma. Epidemiological and clinical profiles were tabulated and analyzed. Prior to conduct the study, the ethical approval was Institutional Ethic Review Committee. Subsequent to this study, each patient provided written informed consent. All the clinically diagnosed hernia patients admitted to the surgical department for surgery were enrolled. The inclusion criteria include primary inguinal hernia patients with elective cases of age above 16 years and shown a willingness to the informed consent form. Patients with previous inguinal hernia, previous surgery, and ventral hernias-like surgical conditions were excluded.

The relevant history, risk factors, demographic facts, examination findings, and clinical data were recorded in a questionnaire, then tabulated and analyzed using SPSS (version 20.0) software. Data analysis was done using statistical methods, which resulted in quantitative results. The outcome of surgery was not a criterion for evaluation.

\section{RESULTS}

The overall mean age of patients was $44.6 \pm 5.6$ with age ranges from 16 years to 72 years. Out of all the operated hernia patients, about 174 (77\%) were male and 52 (23\%) were female as shown in Figure 1. Male to female ratio was 3.34:1. Of the total 226 abdominal wall hernia patients, $202(89.4 \%)$ patients were operated on. The prevalence of emergency-based operated hernia patients was 40 $(19.8 \%)$. One of the most common operated abdominal wall hernia was groin hernia in 162 (80.4\%) followed by umbilical, epigastric, paraumbilical, and rarer hernia with respective prevalence $20(9.9 \%), 8(3.9 \%), 10(4.9 \%)$, and $2(0.9 \%)$ as shown in Table 1 and Figure 2. Ascites, prostatic problems, chronic cough, previous surgeries, chronic constipation, and obesity were the different predisposing factors. The prevalence of predisposing factors were ascites in $3(1.3 \%)$, prostatic problems 46 (20.4\%), chronic cough $113(50 \%)$, previous surgeries 20 (8.8\%), chronic constipation $41(18.4 \%)$, and obesity 3 (1.3\%) as shown in Table-2 and Figure-3.

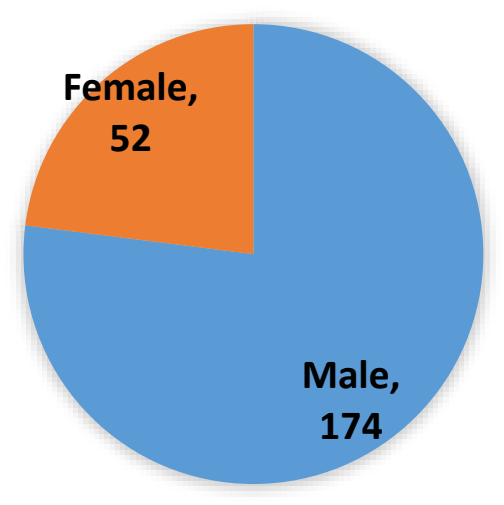

Figure-1 Gender distribution ( $\mathrm{n}=226)$

Table-1 Prevalence of different types of hernia $(n=202)$

\begin{tabular}{|l|l|l|}
\hline $\begin{array}{l}\text { Abdominal Wall Hernia } \\
\text { Types }\end{array}$ & Frequency $\mathrm{n}$ & Percentage \% \\
\hline Groin hernia & 162 & 80.4 \\
\hline Umbilical Hernia & 20 & 9.9 \\
\hline Epigastric & 8 & 3.9 \\
\hline Paraumbilical & 10 & 4.9 \\
\hline Rarer hernia & 2 & 0.9 \\
\hline Total & 202 & 100 \\
\hline
\end{tabular}

Table-2 Prevalence of Predisposing Factors $(n=226)$

\begin{tabular}{|l|l|l|}
\hline Predisposing Factors & Frequency $\mathrm{n}$ & Percentage $\%$ \\
\hline Ascites & 3 & 1.3 \\
\hline Prostatic Problems & 46 & 20.4 \\
\hline Chronic Cough & 113 & 50 \\
\hline Previous Surgeries & 20 & 8.8 \\
\hline Chronic Constipation & 41 & 18.4 \\
\hline Obesity & 3 & 1.3 \\
\hline Total & 226 & 100 \\
\hline
\end{tabular}

Figure-2 Prevalence of different types of Abdominal Wall Hernia $(n=202)$ 


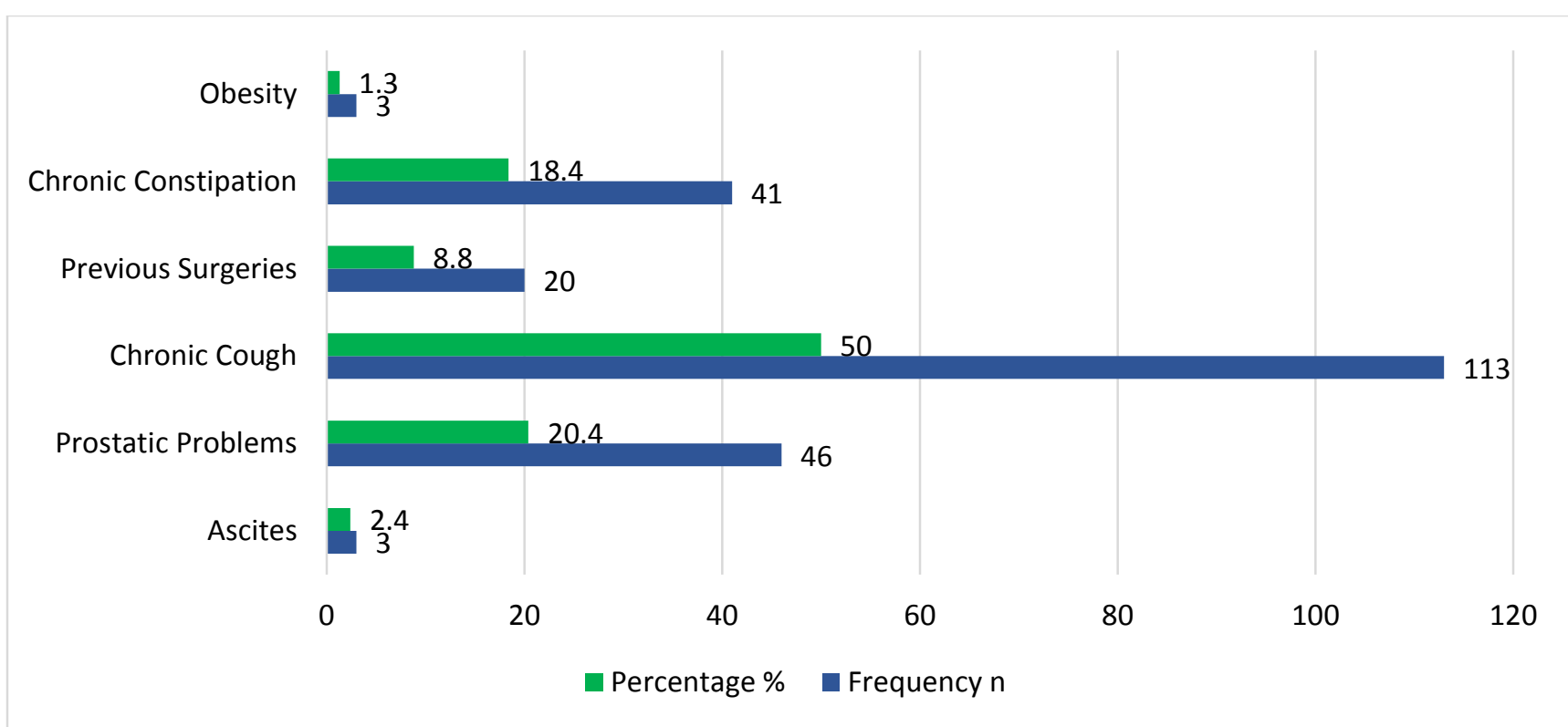

Figure 3 Prevalence of Predisposing Factors $(n=226)$

\section{DISCUSSION}

This epidemiological observational study sought to identify the clinical manifestations and various types of etiological factors for abdominal wall hernia. The present study was also a comprehensive profile study about the impact of available surgical preferences, various comorbidities and outcomes in a tertiary care setting. The research was conducted in Pakistan, where mostly for hernia repair is carried out with laparoscopic procedures. Inguinal hernias are more common in lower socioeconomic groups [13-15]. It is very well reflected in this study, where the maximum patients were from the low, middle, and upper socioeconomic categories. Our study found that inguinal hernia increased with age, but congenital inguinal hernia was found in many patients ( $<19$ years). According to the literature, the inguinal hernia usually diagnosed in patients of age 40 to 60 years [16]. Males were more affected irrespective of the patient's age. Males to female ratio was 3.34:1 ratio. Based on previous research, it has been found that epigastric, femoral, and incisional hernia is more common in females who for various reasons delay their presentation.

In a rural population of Pakistan, delays in hernia presentation to seek hospital care are due to operative intervention phobia, social inhibition, lack of family responsibility, and medical issues concealing tendency. Males are at risk due to chronic cough, preformed strenuous sacs, and activities [17]. Males, too, are prone to arriving late due to their surgical phobia and job loss, putting financial strain. These variables were analogous to those found in other developing countries [18]. The most common risk factors in our study population were older age and smoking. Other common risk factors for the development of inguinal hernia in our study were a history of prostatism, heavy weight lifting, and constipation which correlates with the study of Robindera Kour et al. [19] and some other studies [20, 21]. Malviya et al. [22] found smoking to be an independent risk factor for hernia development in $30.6 \%$ of their study participants. However, family history appears to be an independent risk factor in the etiopathogenesis [23], but only $5 \%$ of the study population had a positive family history.

A study conducted in the United States by Constance et al. [24] discovered that inguinal hernia was associated with older age, chronic cough, obesity, greater height, and rural residence, and this was supported by many other studies such as Lau $\mathrm{H}$ et al. [25] and Junge $\mathrm{K}$ et al. [26], which demonstrated that family history is an important predictor of the development of inguinal hernias and recurrent hernias. Other risk factors mentioned included chronic cough, prostatic hypertrophy, chronic constipation, and chronic diabetes [27].

In our study, the majority of inguinal hernia patients presented late to their health care provider, which is similar to the situation in other developing countries [28]. This results in the appearance of long-standing inguinal hernias. The most common presenting symptoms were a lump and pain, with increased pain as a sign of obstruction. Because of a lack of awareness, fear of surgery, and financial constraints, the majority of our patients presented late to health care providers, which is comparable to other developing countries [29]. Inguinal hernias are more common on the right side, as demonstrated in our study, and this is consistent with previous research. It has been proposed that this is because the right testis descends later, resulting in a higher incidence of failure of closure of the processus vaginalis. [30]

In our study, the most common risk factors for the development of inguinal hernia were strenuous activity such as weight lifting, as well as coexisting benign prostatic hyperplasia and smoking. Increased intra-abdominal pressure has long been suspected as a factor in the pathogenesis of inguinal hernia, but there is little quantitative evidence to support this theory. Constance et al.[31] found no association with factors such as physical activity, constipation, chronic cough, and chronic 
obstructive pulmonary disease that could exert an effect via such a mechanism. A previous study conducted in Spain investigated the increasing risk of inguinal hernia and found that physical exertion, recreational activities, and occupational activities had increased the prevalence of inguinal hernia [32]. In contrast, current sport and physical activities reduce the chances of abdominal wall hernia. Intra-abdominal pressure had no association with an inguinal hernia but might cause constipation which is associated with hernia as reported in a previous study [33].

\section{CONCLUSION}

Our study found that the male population is more susceptible to abdominal wall hernia compared to the female population. Low socioeconomic status with major risk factors such as constipation, old age, lifting heavy objects, smoking, surgery phobia, homeopathy medicine treatment were the main factors for increased rate of morbidity and mortality.

\section{REFERENCES}

1. Pandya B, Huda T, Gupta D, Mehra B, Narang R. Abdominal Wall Hernias: An Epidemiological Profile and Surgical Experience from a Rural Medical College in Central India. The Surgery Journal. 2021 Jan;7(01):e41-6.

2. Kabir AA, Bahar MA, Haque MF, Farooqui MO, Babul M, Akter MS Akhter K, Shahidul M. Evaluation of clinico-epidemiological profile and associated risk factors of inguinal hernia. A Prospective observational study. International Journal of Surgery. 2020;4(3):31-6.

3. Kumar P, Manchanda V, Sengar M. Anterior abdominal wall defects: Demographic and clinical profile and outcome at a tertiary care hospital. Journal of Neonatal Surgery. 2021 Apr 20;10:20-

4. Kaufmann R, Isemer FE, Strey CW, Jeekel J, Lange JF, Woeste G. Non-cross-linked biological mesh in complex abdominal wall hernia: a cohort study. Langenbeck's archives of surgery. 2020 May;405(3):345-52.

5. HerniaSurge Group. International guidelines for groin hernia management. Hernia 2018;22(01):1-165

6. Ayandipo OO, Afuwape OO, Irabor DO, Abdurrazzaaq Al. Adult abdominal wall herniain ibadan. Ann lb Postgrad Med 2015;13(02):94-99

7. ASMA Kabir, M Sharmin, K Akhter, F Akhter, MR Haq, MM Hasan et al. Early Outcome of Lichtenstein Technique for Inguinal Hernia Repair in Tertiary Care Hospital. AKMMC J. 2019; 10(1):62-6.

8. Malviya VK, Sainia TK, Parmar KK, Sharma S. Demographic study in operated patients with inguinal hernia. Surgical Update: Int J surg Orthopedics. 2019; 5:2026.

9. Berthier Nsadi, Olivier Detrt, Willy Arung; Inguinal hernia surgery in developing countries: should laparoscopic repairs be performed? Pan African Medical Journal. 2017; 27:5. doi:10.11604/pamj. 2017; 27.5.12358.

10. Rao S, SinghP, Gupta D,Narang R.Clinicoepidemiologic profile of inguinal hernia in rural medical college in central India. J Mahatma Gandhi Inst Med Sci 2016;21(02):11

11. Deshmukh SN, Varudkar AS, Chopde AV. Clinical study and management of incisional hernias: a prospective monocenter experience. International Surgery Journal. 2017 Apr 22;4(5):1657-61.

12. Mikami DJ, Melvin WS, Murayama MJ, Murayama KM. Impact of minimally invasive surgery on healthcare utilization, cost, and workplace absenteeism in patients with incisional/ventral Hernia (IVH). Surg Endosc. 2017;31 (11):4412-4418. doi:10.1007/s00464017-5488-y.

13. Kubasiak JC, Landin M, Schimpke S, et al. The effect of tobacco use on outcomes of laparoscopic and open ventral hernia repairs: a review of the NSQIP dataset. Surg Endosc. 2017;31(6):2661-2666. doi:10.1007/s00464- 016-5280-4.
14. Nguyen MT, Berger RL, Hicks SC, et al. Comparison of outcomes of synthetic mesh vs suture repair of elective primary ventral herniorrhaphy: a systematic review and meta-analysis. JAMA Surg. 2014;149(5):415-421. doi:10. 1001/jamasurg.2013.5014

15. Holihan JL, Nguyen DH, Nguyen MT, Mo J, Kao LS, Liang MK. Mesh location in open ventral hernia repair: a systematic review and network meta-analysis. World J Surg. 2016;40(1):89-99. doi:10.1007/s00268-015-3252-9.

16. Dietz UA, Winkler MS, Härtel RW, et al. Importance of recurrence rating, morphology, hernial gap size, and risk factors in ventral and incisional hernia classification. Hernia. 2014;18(1):19-30. doi:10.1007/s10029-012-0999-x

17. Howard R, Thompson M, Fan Z, Englesbe M, Dimick JB, Telem DA Costs associated with modifiable risk factors in ventral and incisional hernia repair.JAMA Netw Open. 2019;2(11):e1916330. doi:10.1001/ jamanetworkopen.2019.16330.

18. . Jafri SM, Vitous CA, Dossett LA, et al. Surgeon attitudes and beliefs toward abdominal wall hernia repair in female patients of childbearing age.JAMA Surg 2020;155(6):528-530. doi:10.1001/jamasurg.2020.0099.

19. Robindera Kour, Gurpreet Kour, lqbal Singh, Bimla Bhagat, Dr. KK Gupta. Determining the clinic-demographic profile and associated risk factors of inguinal hernia: A prospective observational study. DOI: https://doi.org/10.33545/surgery.2019.v3.i2c.377.

20. Agrawal SN. A Study of the Demography, Clinical Features and Management of Inguinal Hernia in a Tertiary Care Hospital. J Surgery. 2018;6(1):5.

21. Deshmukh SN, Varudkar AS, Chopde AV. Clinical study and management of incisional hernias: a prospective monocenter experience. International Surgery Journal. 2017 Apr 22;4(5):1657-61.

22. Malviya VK, Sainia TK, Parmar KK, Sharma S. Demographic study in operated patients with inguinal hernia. Surgical Update: Int J surg Orthopedics. 2019; 5:2026.

23. Raam Ratish G. A Prospective study of Abdominal Malignancies Presenting as Abdominal Emergencies in a Tertiary Care Hospital (Doctoral dissertation, Madras Medical College, Chennai).

24. Constance E, James E. Risk Factors for Inguinal Hernia among Adults in the US Population Am J Epidemiol 2017;165:1154-1161. doi: 10.1093/aje/kwm011.

25. Lau H, Fang C, Yuen WK, Patil NG. Risk factors for inguinal hernia in adult males: A case- control study. Surgery. 2016; 141:262-266.

26. Junge K, Rosch R, Klinge U, Schwab R, Peiper C, Binnebosel M et al. - Risk factors related to recurrence in inguinal hernia repair: a retrospective analysis. Hernia. 2016; 10:309-315.

27. Sazhin A, Zolotukhin I, Seliverstov E, Nikishkov A, Shevtsov Y, Andriyashkin A, Tatarintsev A, Kirienko A. Prevalence and risk factors for abdominal wall hernia in the general Russian population. Hernia. 2019 Dec;23(6):1237-42.

28. Salamone G, Licari L, Guercio G, Campanella S, Falco N, Scerrino G, Bonventre S, Geraci G, Cocorullo G, Gulotta G. The abdominal wall hernia in cirrhotic patients: a historical challenge. World Journal of Emergency Surgery. 2018 Dec;13(1):1-6.

29. Gogna S, Latifi R, Policastro A, Prabhakaran K, Anderson P, Con J, Choi J, Samson DJ, Butler J. Complex abdominal wall hernia repair with biologic mesh in elderly: a propensity matched analysis. Hernia. 2020 Jun;24(3):495-502.

30. Clark ST, Malietzis G, Grove TN, Jenkins JT, Windsor AC Kontovounisios C, Warren OJ. The emerging role of sarcopenia as a prognostic indicator in patients undergoing abdominal wall hernia repairs: a systematic review of the literature. Hernia. 2020 Apr 16:1-0.

31. Sneiders D, Yurtkap Y, Kroese LF, Kleinrensink GJ, Lange JF, Gillion JF. Risk factors for incarceration in patients with primary abdominal wall and incisional hernias: a prospective study in 4472 patients. World journal of surgery. 2019 Aug:43(8):1906-13.

32. López-Cano M, Quiles MT, Pereira JA, Armengol-Carrasco M, Arbós Vía MA. Complex abdominal wall hernia repair in contaminated surgical fields: factors affecting the choice of prosthesis. The American Surgeon. 2017 Jun 1;83(6):583-90.

33. Tubre DJ, Schroeder AD, Estes J, Eisenga J, Fitzgibbons RJ. Surgical site infection: the "Achilles Heel" of all types of abdominal wall hernia reconstruction. Hernia. 2018 Dec;22(6):1003-13. 